Supporting Information for:

\title{
Synthesis and Characterization of Functionalized Mesoporous Silica by Aerosol-Assisted Self-Assembly
}

Xiangling $\mathrm{Ji}^{1,3}$, Qingyuan $\mathrm{Hu}^{1}$, J. Eric Hampsey ${ }^{1}$, Xuepeng Qiu², Lianxun Gao ${ }^{2}$, Jibao $\mathrm{He}^{3}$, Yunfeng $\mathrm{Lu}^{1 *}$

${ }^{1}$ Department of Chemical and Biomolecular Engineering, Tulane University, New Orleans, LA 70118, USA

${ }^{2}$ Polymer Engineering Laboratory, Changchun Institute of Applied Chemistry, Chinese Academy of Sciences, Changchun 130022, China

${ }^{3}$ Central Instrumental Facilities, Tulane University, New Orleans, LA 70118, USA

* To whom the corresponding should be addressed. Phone: (504) 865-5827, Fax: (504) 865-6744, email: ylu @ tulane.edu 


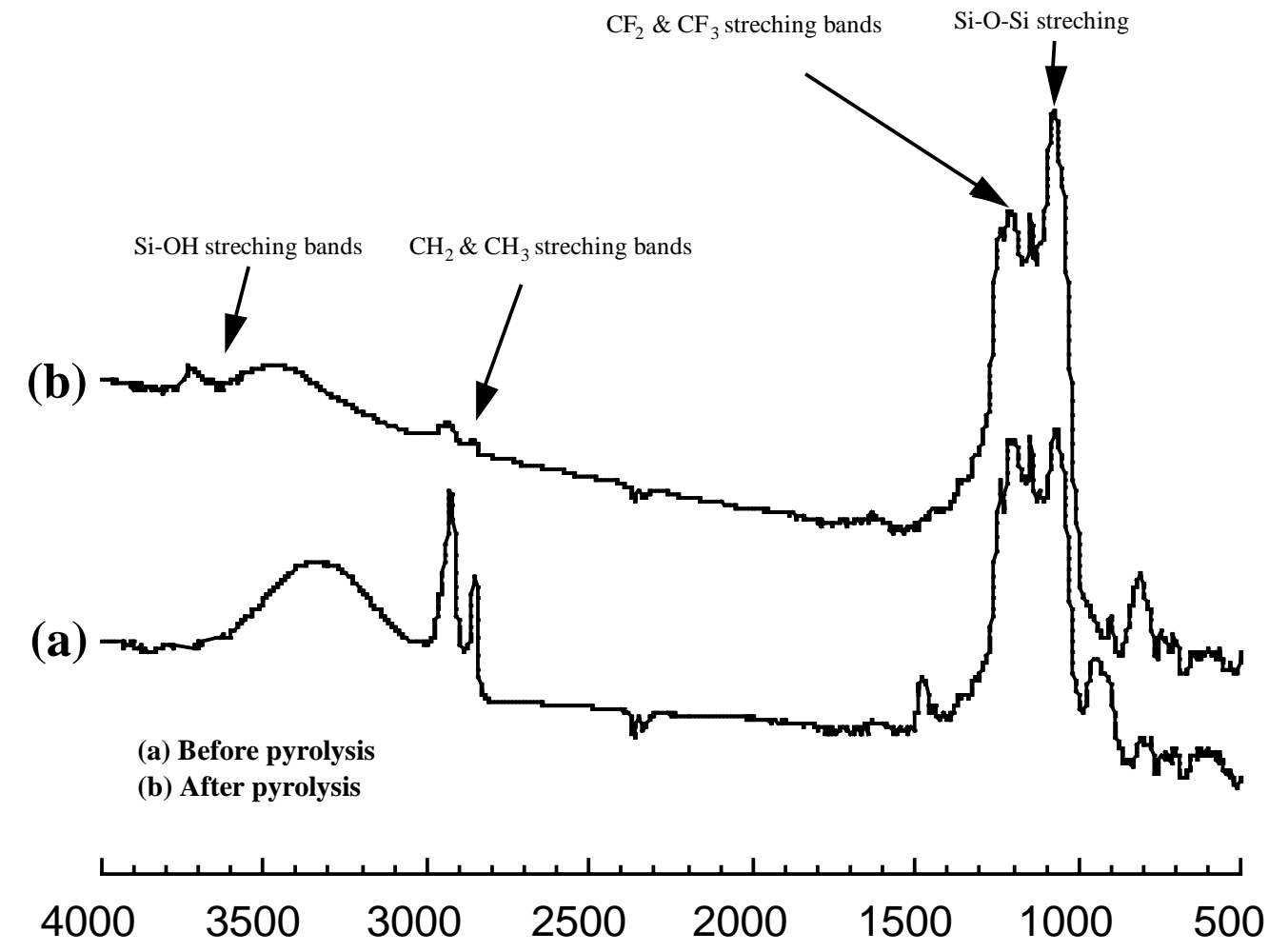

Figure S1. FT-IR spectra of the TFTS-modified silica particles before and after heat treatment at $350{ }^{\circ} \mathrm{C}$ for $5 \mathrm{hr}$ in a nitrogen atmosphere. 


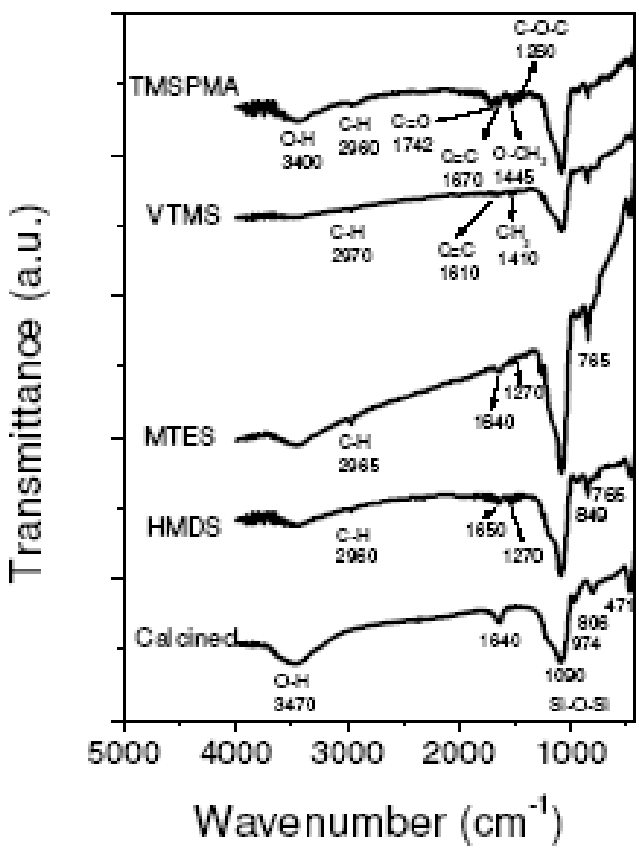

(a)

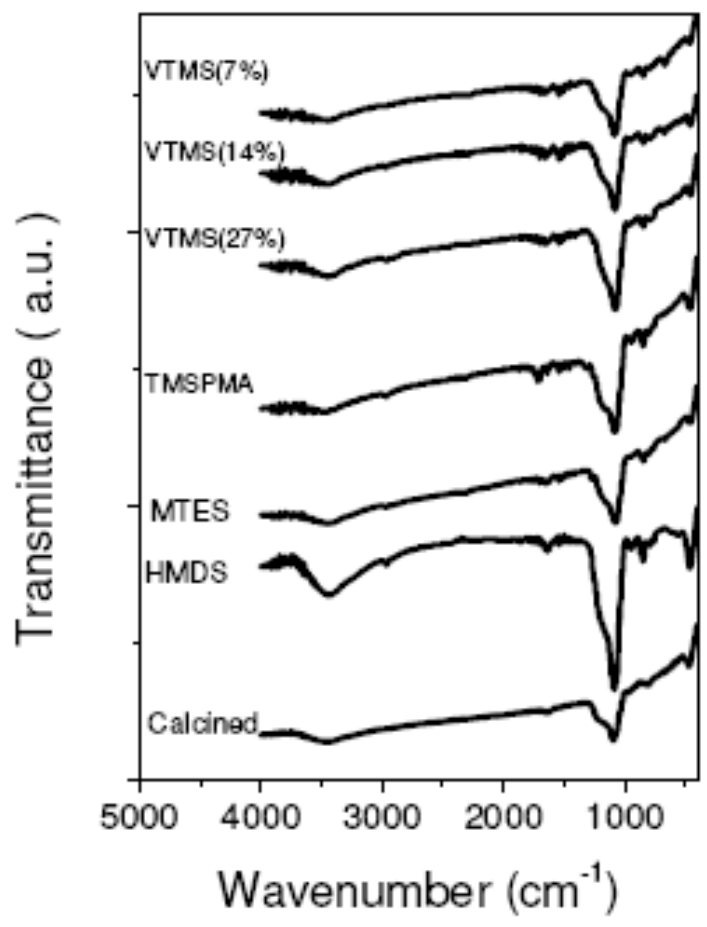

(c)

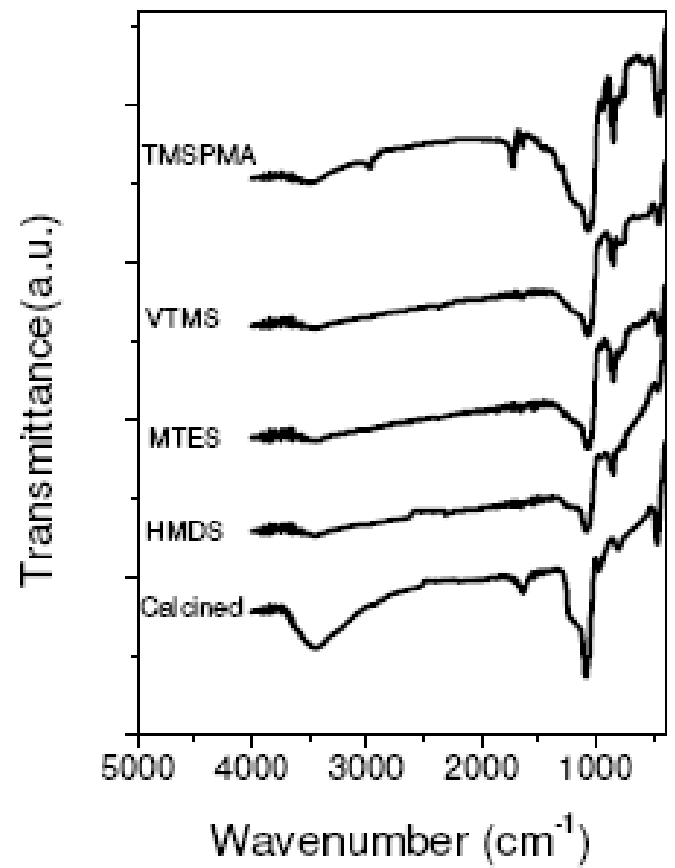

(b)

Figure S2. FT-IR spectra for organic functionalized silica particles, (a) Brij-56, (b) CTAB, (c) P123 as a template. 


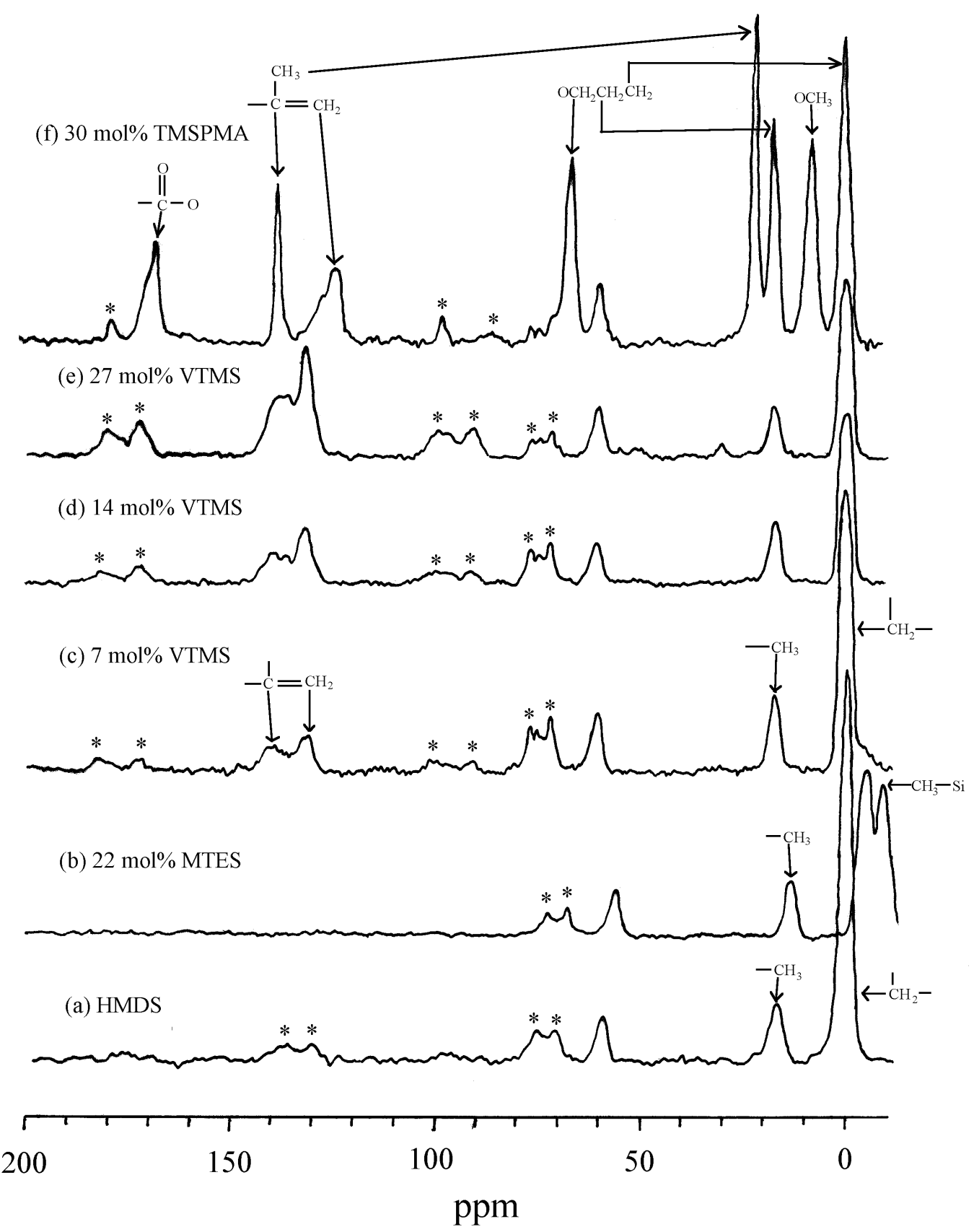

Figure S3. Solid-state ${ }^{13} \mathrm{C} \mathrm{CP/MAS} \mathrm{NMR} \mathrm{spectra,} \mathrm{(a)} \mathrm{HMDS,} \mathrm{(b)} 22$ mol\% MTES, (c) 7 mol\% VTMS, (d) 14 mol\% VTMS, (e) 27 mol\% VTMS, (f) 30 mol\% TMSPMA, * spinning side bands. 


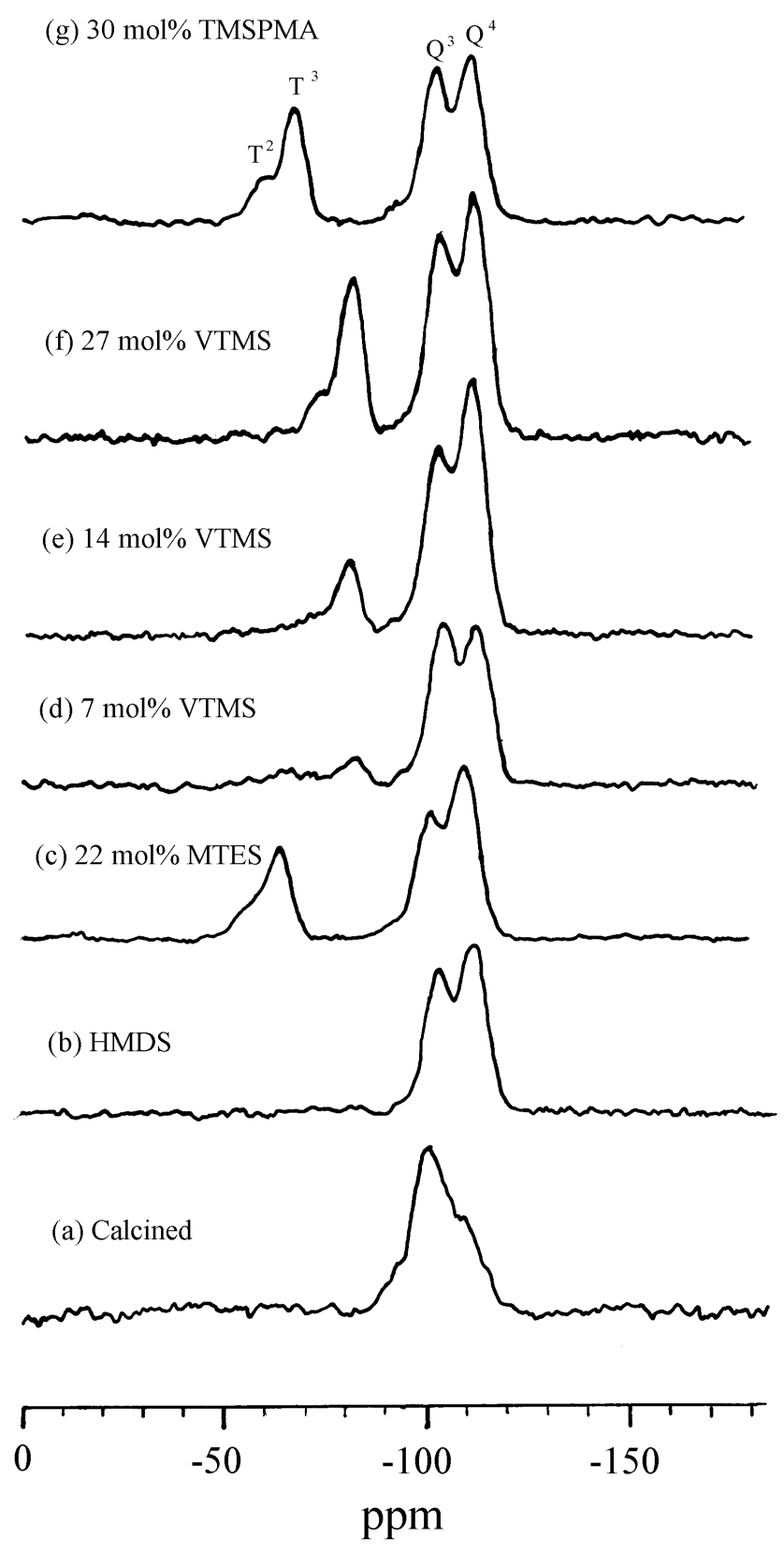

Figure S4. Solid-state ${ }^{29} \mathrm{Si}$ MAS NMR spectra, (a) calcined at $4500^{\circ} \mathrm{C}$ for $4 \mathrm{~h}$, (b) HMDS, (c) 22 mol\% MTES, (d) 7 mol\% VTMS, (e) 14 mol\% VTMS, (f) 27 mol\% VTMS, (g) 30 mol\% TMSPMA. (The cross-polarization (CP) contact time used in this study was $8 \mathrm{~ms}$ ) 


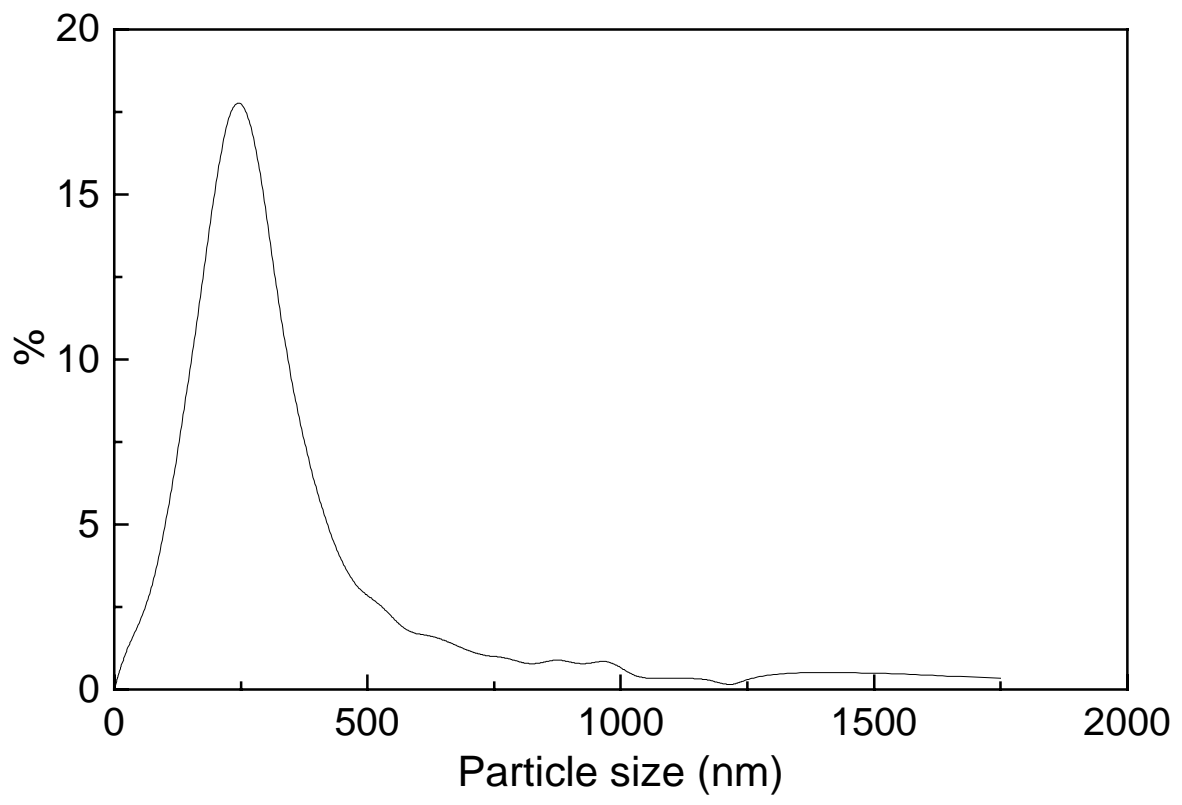

Figure S5 Particle size distribution of samples using P123 as a template 